\title{
New-Onset Dyspnea and Right Heart Failure
}

\author{
Kevin H. Smith Eric S. Bensadoun \\ Division of Pulmonary and Critical Care Medicine, University of Kentucky, Lexington, Ky., USA
}

A 65-year-old male was admitted to hospital with nausea, vomiting and dehydration. He had been diagnosed 6 months earlier with adenocarcinoma of the prostate (Gleason grade $3+4)$ and had completed a course of pelvic radiation 6 weeks prior to admission. He also had a history of rheumatoid arthritis treated with methotrexate, which had been stopped 2 weeks prior to admission due to pancytopenia and elevated liver enzymes.

Upon examination, his vital signs were normal and his physical exam was unremarkable. His laboratory data on admission revealed a hemoglobin of $11.2 \mathrm{~g} / \mathrm{dl}$ and a platelet count of 90,000. Serum electrolyes, glucose, blood urea nitrogen and creatinine were normal. The electrocardiogram was normal and a chest X-ray was unremarkable.

During his admission, his hematocrit and platelet continued to drop and he was noted to have evidence of hemolysis on his peripheral blood smear with an elevated serum $\mathrm{LDH}$ of 12,000 U/l. A presumptive diagnosis of thrombotic thrombocytopenic purpura was made and plasmapheresis was started. After 2 days of plasmapheresis, the platelet count had stabilized; however, the patient began to complain of dyspnea and his oxygen saturation was noted to be $85 \%$ on room air. A CT angiogram of the chest (see fig. 1) was performed and was negative for pulmonary embolism. An echocardiogram revealed right ventricular enlargement with decreased right ventricular systolic function with an estimated right ventricular systolic pressure of $74 \mathrm{~mm} \mathrm{Hg}$. Two months earlier, the patient had an echocardiogram that was normal. The pa-

\section{KARGER}

E-Mail karger@karger.com www.karger.com/res

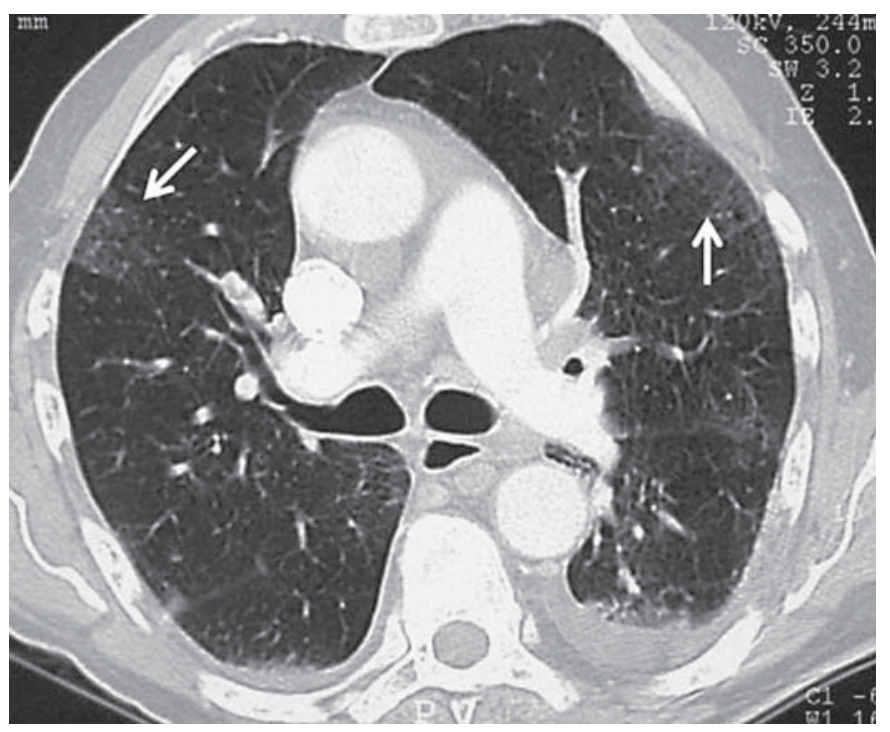

Fig. 1. CT angiogram of the chest demonstrates a few scattered areas of faint peripheral ground-glass opacity (arrows) without evidence of pulmonary embolism. A small left pleural effusion is also seen.

tient's hypoxemia worsened and he was transferred to the intensive care unit. A pulmonary artery catheter was inserted which revealed a pulmonary artery pressure of $64 / 24 \mathrm{~mm} \mathrm{Hg}$, a pulmonary capillary wedge pressure of $8 \mathrm{~mm} \mathrm{Hg}$, and a cardiac index of $3.7 \mathrm{l} / \mathrm{min} / \mathrm{m}^{2}$.

What is your diagnosis? (c) 2013 S. Karger AG, Basel

0025-7931/13/0873-0249\$38.00/0
Eric S. Bensadoun, MD, MPH

Division of Pulmonary and Critical Care Medicine, University of Kentucky 740 South Limestone, L543 Kentucky Clinic

Lexington, KY 40536-0298 (USA)

E-Mail ebens0@uky.edu 


\section{Diagnosis: Tumor Emboli to the Lungs from Metastatic Adenocarcinoma of the Prostate}

A sample of blood was aspirated from the distal port of the pulmonary artery catheter in a wedged position. This was sent for cytological evaluation, which showed a branching aggregate of malignant cells consistent with metastatic adenocarcinoma (see fig. 2). These cells stained positive for prostate-specific antigen on immunohistochemistry, confirming a diagnosis of tumor emboli to the lungs from metastatic prostate cancer.

There is a spectrum of disease associated with tumor emboli, and tumor involvement of the pulmonary vasculature can manifest in a variety of ways. Large, proximal tumor embolization can result in acute decompensation such as that seen with massive thromboembolism. In contrast, microvascular tumor embolization manifests with progressive, often insidious dyspnea and respiratory failure. Microscopic tumor emboli become lodged in the pulmonary capillary bed and cause obstruction of pulmonary flow via one of two mechanisms: the mechanical occlusion of vessels with tumor cells or activation of a coagulation cascade at the tumor cell surface resulting in the production of occluding microthrombi and local vascular remodeling, referred to as 'pulmonary tumor thrombotic microangiopathy' [1-4]. Both of these mechanisms play a role in the pathogenesis of pulmonary hypertension and the associated right-sided heart failure that can be seen with tumor embolism.

The overall incidence of tumor emboli among patients with solid organ malignancies ranges from 0.2 to $2.4 \%$ based on retrospective autopsy studies $[1,5]$, although in patients with choriocarcinoma and carcinoma of the breast, liver, stomach or kidneys, the collective incidence can be as high as $26 \%$ [1]. Most reported cases of tumor emboli have been associated with carcinoma of the breast, lung, stomach, liver or prostate; however, isolated cases with a variety of other malignancies have also been reported $[1,6]$.

The nonspecific presentation and the lack of a sensitive and specific noninvasive test make the antemortem diagnosis of tumor embolism very challenging. Progressive, subacute dyspnea is the most common clinical presentation. Hypoxemia is usually present; however, the signs of pulmonary hypertension and right heart failure are only seen in $15-20 \%$ of patients $[1,6]$. Chest X-rays and ECG are typically unremarkable. Echocardiogram may show evidence of pulmonary hypertension while CT of the chest may sometimes show the peripheral pulmonary arteries to have a dilated and beaded appearance $[1,6]$. Pulmonary

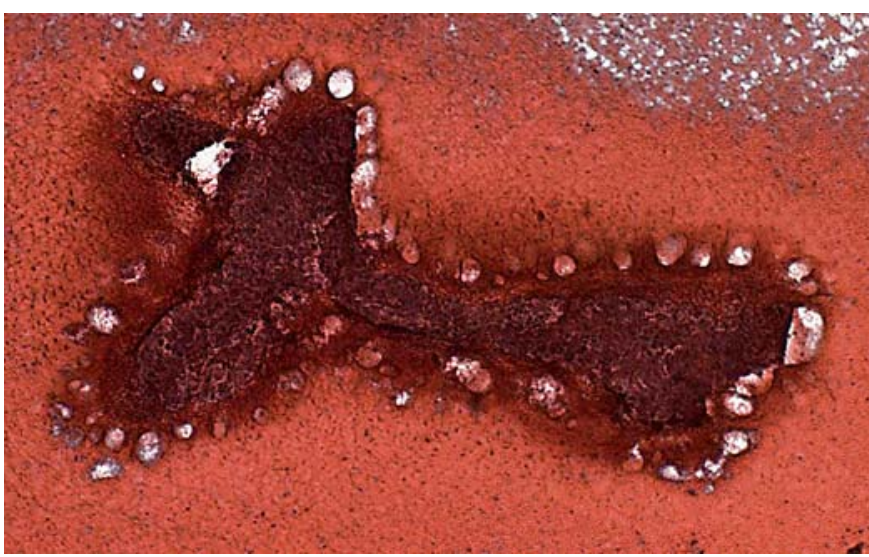

Fig. 2. Cytology specimen from the PA catheter aspirate demonstrates a branching aggregate of malignant cells forming a vascular cast of a small pulmonary artery $(\mathrm{PAP} \times 40)$. The cytological features and immunohistochemical stains were consistent with a diagnosis of tumor emboli from metastatic adenocarcinoma of the prostate.

angiogram or CT angiogram is insensitive for microscopic tumor emboli and serves only to rule out thromboembolism or make an alternative diagnosis $[1,6]$. The ventilation-perfusion scan can be helpful as it sometimes reveals a 'segmental contour' pattern consisting of numerous small peripheral subsegmental perfusion defects with normal ventilation which may be useful in differentiating tumor emboli from thromboembolism [1]. Another method for the diagnosis of tumor embolism is the cytological examination of pulmonary artery blood. This is performed by aspirating blood from the distal port of a pulmonary artery catheter in the wedged position. The cytology requires expert interpretation as normal pulmonary megakaryocytes can sometimes be mistaken for malignant cells $[7,8]$. The presence of malignant cells within the pulmonary vasculature in the right context makes a strong case for a diagnosis of tumor embolism [1].

In many cases, a definitive diagnosis will require tissue confirmation with an open-lung biopsy [1]. The diagnosis of tumor embolism should be considered in the patient with a history of solid malignancy who has new-onset dyspnea, evidence of pulmonary hypertension and negative diagnostic testing for thromboembolic disease. How aggressively this diagnosis is to be pursued should be mitigated by the risks of the procedures contemplated and the responsiveness of the underlying malignancy, if known, to potential therapy. 


\section{References}

$>1$ Bassiri AG, Haghighi B, Doyle RL, et al: Pulmonary tumor embolism. Am J Respir Crit Care Med 1997;155:2089.

$\checkmark 2$ von Herbay A, Illes A, Waldherr R, Otto HF: Pulmonary tumor thrombotic microangiopathy with pulmonary hypertension. Cancer 1990;66:587-592.

3 Patrignani A, Pucaro A, Calcagnoli F, Mandolesi A, Bearzi I, Ciampani N: Pulmonary tumor thrombotic microangiopathy: the challenge of the antemortem diagnosis. J Cardiovasc Med 2013, E-pub ahead of print.
4 Uraga H, Fugii T, Kurosaki A, et al: Pulmonary tumor thrombotic microangiopathy: a clinical analysis of 30 autopsy cases. Intern Med 2013;52:1317-1323.

5 Sakuma M, Fukui S, Nakamura M, et al: Cancer and pulmonary embolism: thrombotic embolism, tumor embolism, and tumor invasion into a large vein. Circ J 2006;70:744-749.
6 Roberts KE, Hamela-Bena D, Saqi A, et al: Pulmonary tumor embolism: a review of the literature. Am J Med 2003;115:228-232.

7 Masson RG, Krikorian J, Lukl P, et al: Pulmonary microvascular cytology in the diagnosis of lymphangitic carcinomatosis. N Engl J Med 1989;321:71-76.

8 Masson RG, Ruggieri J: Pulmonary microvascular cytology: a new diagnostic application of the pulmonary artery catheter. Chest 1985; 88:908. 